\title{
Group Integral Techniques for the Spinfoam Graviton Propagator
}

\author{
Etera R. Livine* \\ Laboratoire de Physique, ENS Lyon, CNRS UMR 5672, 46 Allée d'Italie, 69364 Lyon, France, and \\ Perimeter Institute, 31 Caroline St. N, Waterloo, ON N2L 2Y5, Canada. \\ Simone Speziale St $^{\dagger}$ \\ Perimeter Institute, 31 Caroline St. N, Waterloo, ON N2L 2Y5, Canada.
}

\begin{abstract}
We consider the recent proposal [1] for the extraction of the graviton propagator from the spinfoam formalism. We propose a new ansatz for the boundary state, using which we can write the propagator as an integral over $\mathrm{SU}(2)$. The perturbative expansion in the Planck length can be recast into the saddle point expansion of this integral. We compute the leading order and recover the behavior expected from low-energy physics. In particular, we prove that the degenerate spinfoam configurations are suppressed.
\end{abstract}

\section{INTRODUCTION}

Recently [1, 2, 3, 3], a proposal has appeared for the computation of the 2-point function of quantum gravity within the spinfoam formalism [ $[$ ], a candidate covariant approach to a non-perturbative quantisation of General Relativity (GR). This proposal offers a possibility to study the semiclassical limit of spinfoams and define the perturbative expansion in the Planck length $\ell_{\mathrm{P}}$, arguably the major open question within this approach. This is very interesting, for two different reasons: on the one hand, to check the correctness of the low-energy limit of spinfoams; on the other hand, to address the possibility of curing the non-renormalisability of the conventional perturbative expansion via background-independent methods.

In 1] (see also [5]), the proposal was applied to the Barrett-Crane (BC) spinfoam model for four dimensional (4d) Riemannian GR, and it was shown that the 2-point function, or graviton propagator, indeed had the correct $1 /|x-y|^{2}$ leading order behavior in the large scale limit. However, there are some assumptions behind this result that deserve a more careful treatment. In particular, the BC model is plagued by degenerate configurations. This has so far cast some doubts on the viability of the proposal, especially because there is still no support from the numerical analysis, due to the high complexity of the vertex amplitude.

In this work, we prove that the degenerate configurations do not affect the leading order. In doing so, we modify the ansatz for the boundary state, following the 3 d investigation appeared in [4]. For the 4-simplex spinfoam contribution, the new boundary state allows us to write the 2-point function as an integral over $\mathrm{SU}(2)$. This is our first result. In the large $j$ limit, we evaluate this integral in a saddle point approximation, proving the $1 /|x-y|^{2}$ behavior of the leading order. The degenerate configurations can be correctly neglected because they correspond to saddle points which are not absolute minima. This is our second result. Thanks to the integral expression, which involves no sums, the numerical analysis is strongly simplified. Indeed, the leading order result is fully supported by numerical analysis [14].

Concerning the full perturbative expansion in $\ell_{\mathrm{P}}$, this can be computed from higher orders of the saddle point approximation as well as from the contribution of other spinfoams. We do not attempt here their evaluation, which is rather complicate. Notice however that higher orders have been studied in a 3d toy model [3, 4]; remarkably, interesting modifications to the conventional expansion arise, due to the microscopical quantum geometry described by spinfoams. This makes the whole approach very interesting, and pushes towards the calculation of these corrections in the $4 \mathrm{~d}$ case. We expect that having recast the $\ell_{\mathrm{P}}$ expansion into the saddle point expansion will help future work.

This paper is organised as follows. In the next Section, we briefly recall properties of the BC vertex amplitude which we need in the rest of the paper. In Section III, we review the construction of the 2-point function and the results of [1]. In Section IV] we introduce the new boundary state and discuss its properties. In Section $\mathrm{V}$, we show how the new boundary state allows to write the 2-point function as an integral over SU(2). In Section VI] we study

\footnotetext{
*elivine@perimeterinstitute.ca

† sspeziale@perimeterinstitute.ca
} 
the saddle point approximation to this integral, and show that the leading order behaves as expected from low-energy physics. In the final Section, we discuss possible further developments.

Throughout the paper, we use units $\ell_{\mathrm{P}}=1$.

\section{SPINFOAM AMPLITUDES: EVALUATION OF RELATIVISTIC SPIN NETWORKS}

In this Section we recall basic facts about the spinfoam amplitudes, the quantities encoding the dynamics of quantum gravity. This gives us the opportunity to fix our notation and to pinpoint features which will be crucial in the following. We consider here the spinfoam amplitude for the $\mathrm{BC}$ model of $4 \mathrm{~d}$ Riemannian quantum gravity; this is given by the evaluation of a relativistic spin network 7] with group $\operatorname{Spin}(4)$, namely the double cover of $\mathrm{SO}(4)$. The relativistic spin network is defined by a graph $\Gamma$ together with the assignment of a $\operatorname{Spin}(4)$ group element $G_{n}$ to each node $n$ of $\Gamma$ and a simple irreducible representation (irrep) $J_{l}$ to each link $l$ of $\Gamma$. Using the homomorphism $\operatorname{Spin}(4)=\mathrm{SU}(2)_{L} \times \mathrm{SU}(2)_{R}$, the irreps of $\operatorname{Spin}(4)$ are labelled by two half-integers, say $(j, k)$, corresponding to the irreps of the two $\mathrm{SU}(2)$ sectors; then the simple representations are such that they induce the same $\mathrm{SU}(2)$ representation in the left and right sectors, namely $J_{l}=\left(j_{l}, j_{l}\right)$. Furthermore, let us note here that the scalar Casimir of $\operatorname{Spin}(4)$ satisfies $C_{\operatorname{Spin}(4)}^{2}(j, k)=4\left[C^{2}(j)+C^{2}(k)\right]$, where $C^{2}(j)$ is the $\mathrm{SU}(2)$ Casimir.

Using the above homomorphism, each group element decomposes as the product of two left and right rotations $G=g_{L} g_{R}$, and the evaluation reads []],

$$
\mathcal{E}_{\Gamma} \equiv \int_{\operatorname{Spin}(4)} \prod_{n} d G_{n} \prod_{l} \mathcal{K}_{J_{l}}\left(G_{s(l)}^{-1} G_{t(l)}\right)
$$

where $s(l)$ and $t(l)$ denote the source and target node of the link $l$. The kernel $\mathcal{K}_{J}(G)$ is the matrix element of $G$ on the $\mathrm{SU}(2)$-invariant vector $|J, 0\rangle$ in the $J$ representation. Here $\mathrm{SU}(2)$ is the diagonal rotation group, corresponding to the subgroup of $3 \mathrm{~d}$ rotations. We conveniently parametrise $\mathrm{SU}(2)$ group elements as

$$
g(\phi, \hat{n})=\cos \phi \mathbb{1}+i \sin \phi \hat{n} \cdot \vec{\sigma}, \quad \phi \in[0, \pi] .
$$

Consequently, the characters are given by $\chi_{j}(g)=\frac{\sin d_{j} \phi}{\sin \phi}$, and the Haar measure is $d g=\frac{1}{2 \pi^{2}} \sin ^{2} \phi d^{2} \Omega(\hat{n}) d \phi$.

The invariant vector is easily expressed in term of left/right components:

$$
|J, 0\rangle=\frac{1}{\sqrt{d_{j}}} \sum_{m}(-1)^{j-m}|j, m\rangle_{L}|j,-m\rangle_{R}=\frac{1}{\sqrt{d_{j}}} \sum_{m}|j, m\rangle_{L}{ }_{R}\langle j, m|,
$$

where $d_{j}=2 j+1$ is the dimension of the $\mathrm{SU}(2)$ representation of spin $j$. Then it is straightforward to realize that the $\mathrm{SU}(2)$ invariant kernel $\mathcal{K}_{J}$ is simply the $\mathrm{SU}(2)$ character:

$$
\langle J, 0|G| J, 0\rangle=\left\langle J, 0\left|g_{L} g_{R}\right| J, 0\right\rangle=\frac{1}{d_{j}} \sum_{m}\left\langle j, m\left|g_{L} g_{R}^{-1}\right| j, m\right\rangle=\frac{1}{d_{j}} \chi_{j}\left(g_{L} g_{R}^{-1}\right) .
$$

Finally, using the invariance of the Haar measure $d G=d g_{L} d g_{R}$ under left and right multiplication, it is easy to prove that the relativistic spin network evaluation is actually a $3 \mathrm{~d}$ object regarding only integrals over $\mathrm{SU}(2)$ :

$$
\mathcal{E}_{\Gamma}=\int_{\mathrm{SU}(2)} \prod_{n} d g_{n} \prod_{l} \frac{1}{d_{j_{l}}} \chi_{j_{l}}\left(g_{s(l)}^{-1} g_{t(l)}\right),
$$

In particular, the vertex amplitude for the $\mathrm{BC}$ model is obtained for $\Gamma$ given by a 4 -simplex, and this gives the $\{10 j\}$ symbol for the recoupling theory of $\mathrm{SU}(2)$,

$$
\{10 j\}=\int_{\mathrm{SU}(2)} \prod_{n} d g_{n} \prod_{l} \frac{1}{d_{j_{l}}} \chi_{j_{l}}\left(g_{s(l)}^{-1} g_{t(l)}\right) .
$$


Let us point out that the $\{10 j\}$ symbol is defined up to a normalisation. This creates an ambiguity in the definition of the $\mathrm{BC}$ model. The standard normalisation found in the literature is (6) without the $1 / d_{j}$ factors. Notice also that another natural choice for the kernel is $d_{j}^{2} \mathcal{K}_{J}=d_{j} \chi_{j}$, instead of $\chi_{j} / d_{j}$, as it normalises the convolution product:

$$
\int_{\operatorname{Spin}(4)} d G\left(d_{j}^{2} \mathcal{K}_{J}\left(H G^{-1}\right)\right)\left(d_{j}^{2} \mathcal{K}_{J}\left(G K^{-1}\right)\right)=d_{j}^{2} \mathcal{K}_{J}\left(H K^{-1}\right)
$$

These normalisation issues do not modify the computations below, namely the leading order of the graviton correlation computed using a single 4-simplex. We nevertheless mention them as they will most likely affect the higher order corrections and become essential when considering configurations with many 4-simplices.

\section{Geometrical interpretation of the $\{10 j\}$ symbol}

The $\{10 j\}$ symbol admits a geometrical interpretation, associated to the structure of a 4 -simplex, which will be important in the following. The key fact is that it can be written as an integral over ten $\mathrm{SU}(2)$ angles $\phi_{l} \in[0, \pi]$,

$$
\{10 j\}=\int d \mu\left[\phi_{l}\right] \prod_{l} \frac{1}{d_{j_{l}}} \chi_{j_{l}}\left(\phi_{l}\right)
$$

where the measure takes into account that the angles come from the vertex group elements $g_{v}$ through the relation, $\cos \phi_{l}=\frac{1}{2} \operatorname{tr}\left(g_{s(l)}^{-1} g_{t(l)}\right)$. Let us introduce the notation $(I J)$ for $l$ linking the nodes $I$ and $J$, such that $\phi_{I J} \equiv \phi_{l}$, with the convention $\phi_{I I}=0$. The above relation imposes a constraint that can be written as the vanishing of the determinant of the $5 \times 5$ Gram matrix $G_{I J}=\cos \phi_{I J}$, as shown in [12]:

$$
d \mu\left[\phi_{l}\right]=\prod_{l} d \phi_{l} \sin \phi_{l} \delta\left(\operatorname{det} G_{I J}\right) .
$$

This constraint has a clear geometrical interpretation: it says that the angles $\phi_{l}$ are the dihedral angles of a certain 4-simplex. Indeed, notice that the spin network induces a dual triangulation which is also a 4 -simplex, with tetrahedra dual to the nodes and triangles dual to the links (see Fig.11). Then the constraint can be translated into the Schläfli identity $\sum_{l} A_{l}(\phi) d \phi_{l}=0$, where $A_{l}$ is the area of the triangle (dual to the link $l$ ) of the geometric 4-simplex, and $\phi_{l}$ its dihedral angle. The areas can be written as derivatives of the Gram matrix, $A_{l}=\kappa \frac{\partial \operatorname{det} G_{I J}}{\partial \phi_{l}}$, where $\kappa$ is a proportionality constant, related to the 4 -volume of the simplex. For more details see the Appendix.

The original group integration in (6) is over 5 copies of the 3 -sphere $\mathcal{S}^{3} \sim \operatorname{Spin}(4) / \mathrm{SU}(2)$ and the 10 angles $\phi_{l}$ are easily related to the 5 original $4 \mathrm{~d}$ unit vectors $\hat{N}_{I} \in \mathcal{S}^{3}$, via $\cos \phi_{I J}=\hat{N}_{I} \cdot \hat{N}_{J}$.

For later use, let us consider the equilateral case when all the dihedral angles are equal, $\phi_{l}=\varphi$. Then the constraint simply reads

$$
\operatorname{det} G_{I J}\left(\phi_{l}=\varphi\right)=(1-\cos \varphi)^{4}(1+4 \cos \varphi),
$$

whose roots are given by the completely degenerate 4 -simplex $\varphi=0$, and the equilateral 4 -simplex $\varphi \equiv \theta=$ $\arccos (-1 / 4)$.

To summarise, two important features emerge from the above discussion:

- The relevant group for 4d Riemannian quantum gravity without matter is $\mathrm{SU}(2)$.

- The angles $\phi$ parametrising SU(2) group elements can be thought of as dihedral angles between the tetrahedra dual to the nodes of the boundary spin network entering the spinfoam amplitude.

Equipped with these considerations, we now proceed to describe how to construct the 2-point function. 


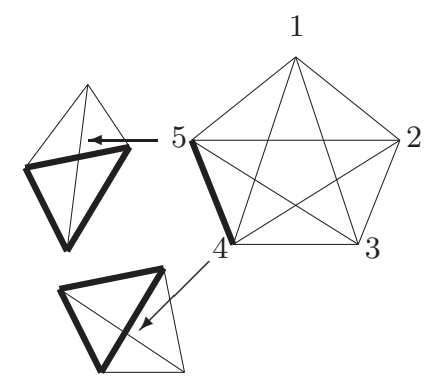

FIG. 1: The 4-simplex (or pentahedral) boundary spin network. We label the nodes $I=1, \ldots 5$. In the dual picture, they are in correspondence with tetrahedra of the boundary triangulation. Two of them are represented. The links $I J$, on the other hand, are dual to triangles. Consider for instance the link 45: this is dual to the triangle shared by the tetrahedra 4 and 5 . Associated with the link 45 is the dihedral angle $\phi_{45}$ between the tetrahedra 4 and 5 .

\section{THE 2-POINT FUNCTION}

In the conventional quantum field theory framework one expands the metric tensor around a given background, typically the Minkowski flat spacetime $\eta_{\mu \nu}$, writing $g_{\mu \nu}=\eta_{\mu \nu}+\ell_{\mathrm{P}} h_{\mu \nu}$; then the 2-point function,

$$
W_{\mu \nu \rho \sigma}(x, y)=\left\langle 0\left|\mathrm{~T}\left\{h_{\mu \nu}(x) h_{\rho \sigma}(y)\right\}\right| 0\right\rangle,
$$

is evaluated in perturbation theory. The leading order, coming from the quadratic term in the action, goes as $1 /|x-y|^{2}$, namely as $1 / p^{2}$ in momentum space. The higher orders in the action give self-energy corrections.

In the spinfoam formalism, the 2-point function (10) can be studied looking at the correlations between fluctuations of geometrical quantities. The fluctuations are defined with respect to a boundary metric which is encoded in a spin network state $s$. The points $x$ and $y$ in (10) can be identified with nodes of $s$, and the directions $\mu$ can be defined using the links of $s$. In particular, since each link of $s$ can be thought of as dual to a triangle in a triangulation of the boundary, it is convenient to project (10) along the normals to these triangles. Namely, using the boundary metric, we contract the indices of (10) with four normal vectors, two belonging to the ( $3 \mathrm{~d}$ region dual to) the node $x$ and two to the node $y$. For each node, we should distinguish the case when we take twice the same normal, from the case when we consider two different normals. In the first case, the contraction with $h_{\mu \nu}$ gives (the fluctuation of) the area of the triangle. In the second case, of the dihedral angle between the two triangles.

In the quantum theory, both areas and dihedral angles are represented by quantum operators. The area operator has spectrum given by twice the square root of the $\mathrm{SU}(2)$ Casimir operator, ${ }^{1} A=2 C(j)=2 j+1$. Analogously, the angle operator can be expressed in terms of $\mathrm{SU}(2)$ Casimir operators, via the recoupling theorem (see for instance [5, 9]) . This is how the spin variables entering the amplitudes are related to geometrical quantities. Since the fundamental variables of the theory are triangle areas and dihedral angles, we compute the 2-point function as the correlation between fluctuations of these quantities around a flat background. Choosing a flat background for the boundary geometry allows us to introduce a (spin) scale parameter, which we call $j_{0}$. We do so by describing the triangulation of the flat boundary in terms of equilateral triangles with areas $A_{0}=2 C\left(j_{0}\right)$. The parameter $j_{0}$ can be used to measure the physical distance between two points on the boundary with respect to the flat background metric.

In the following, we consider only the correlation between the areas, leaving a discussion of the other cases for the conclusive Section. Introducing the normal vectors $n_{a}^{\mu}$ and $n_{a}^{\mu}$ to the triangles $a$ and $b$, the area correlations can be compared with the projections $n_{a}^{\mu} n_{a}^{\nu} n_{b}^{\rho} n_{b}^{\sigma} W_{\mu \nu \rho \sigma}(x, y)$ of the continuum 2-point function. Notice that if the 4-simplex is equilateral, there are only three independent projections [5]; these correspond to the three cases when the triangles $a$ and $b$ are the same, when they share a side, or when the share only a point. Following [1], the area correlations are

\footnotetext{
1 The factor 2 between the triangle area and the Casimir can be understood as follows. Given the two edge vectors $a^{I}, b^{J}$ of the triangle, the Spin(4) Casimir can be identified with the norm of the bivector $B^{I J}=\frac{1}{2}\left(a^{I} b^{J}-b^{I} a^{J}\right)$, namely with the quantity $\frac{1}{2}|a|^{2}|b|^{2} \sin ^{2} \theta \equiv 2 A^{2}$. Therefore $2 A^{2}=C_{\mathrm{Spin}(4)}^{2}(j, j) \equiv 8 C^{2}(j)$, using the homomorphism between $\operatorname{Spin}(4)$ and $\mathrm{SU}(2)$ introduced above. As for the spectrum, let us recall that the Casimir operator is always defined up to an additive constant. Usually one takes this constant to zero, so that its spectrum reads $C^{2}(j)=j(j+1)$. On the other hand, here we take a shift of $1 / 4$, so that $C^{2}(j)=\left(j+\frac{1}{2}\right)^{2}$, to match the results on the asymptotics of the $\{10 j\}$ symbol reported below.
} 
given by

$$
W_{a b}\left(j_{0}\right)=\frac{1}{j_{0}^{4}} \frac{1}{\mathcal{N}} \sum_{s} \Psi_{0}[s] \mathbb{h}\left(j_{a}\right) \mathbb{h}\left(j_{b}\right) \mathcal{A}[s]
$$

where $\mathcal{N}=\sum_{s} \Psi_{0}[s] \mathcal{A}[s]$ is the normalisation, and the factor $1 / j_{0}^{4}$ comes from normalising the projections along the normals.

Let us briefly explain this formula, referring to [5] for a more complete description. The graviton propagator is expressed as a sum over all possible boundary spin networks. This includes a sum over all the the graphs and a sum over all possible assignments of spins to the links of the graphs.

The boundary state $\Psi_{0}[s]$ should represent a coherent semiclassical state of the boundary geometry [5]. In particular, we require that the relative uncertainties of the geometry on this state vanish in the large $j_{0}$ limit, namely

$$
\frac{\left\langle\Psi_{0}\left|\Delta j_{l}\right| \Psi_{0}\right\rangle}{\left\langle\Psi_{0}\left|j_{l}\right| \Psi_{0}\right\rangle} \mapsto 0, \quad \frac{\left\langle\Psi_{0}\left|\Delta \phi_{l}\right| \Psi_{0}\right\rangle}{\left\langle\Psi_{0}\left|\phi_{l}\right| \Psi_{0}\right\rangle} \mapsto 0
$$

for all links $l$ in the boundary spin network.

The quantity $\mathbb{h}\left(j_{a}\right):=C^{2}\left(j_{a}\right)-C^{2}\left(j_{0}\right)$ represents the field insertion $h_{\mu \nu}(x)$ in a Coulomb-like gauge-fixing [3] . We see that it is (one fourth) the fluctuation of the squared area.

The amplitude $\mathcal{A}[s]$ is given by the spinfoam model chosen, and it is a sum over all spinfoams $\sigma$ whose boundary $\partial \sigma$ is given by the spin network $s$. Working with the Barrett-Crane model, we have

$$
\mathcal{A}[s]=\sum_{\substack{\sigma \\ \partial \sigma=s}} \sum_{j_{f}} \prod_{f} d_{j_{f}}^{2} \prod_{e \in \sigma \backslash \partial \sigma} A_{e}\left(j_{f}\right) \prod_{e \in \partial \sigma}\left(A_{e}\left(j_{f}\right)\right)^{\frac{1}{2}} \prod_{v}\{10 j\}
$$

where $v, f$ are respectively the vertices and faces of the spinfoam, and $j_{f} \equiv j_{l}$ whenever a face $f$ intersects the boundary forming a link $l$. The vertex amplitude is given by the $\{10 j\}$ symbol introduced in the previous Section. Notice that we left unspecified the edge amplitude $A_{e}\left(j_{f}\right)$. This reflects the normalisation ambiguity of the $\{10 j\}$ symbol, and different choices lead to different versions of the $\mathrm{BC}$ model ${ }^{2}$. However we expect changes in the edge amplitudes not to affect the leading order of the graviton correlations $W_{a b}$, in analogy with the $3 \mathrm{~d}$ case [4]. They will nevertheless affect the higher order corrections, allowing to distinguish and discriminate the different choices of $A_{e}$. Below we will choose a particular edge amplitude which simplifies the calculations of the leading order.

The expression (11) is the full graviton propagator. This can be evaluated in perturbation theory. The parameter of the conventional perturbative expansion is the Planck length $\ell_{\mathrm{P}}$. By dimensional analysis, it appears (squared) in front of $j_{0}$. We are then led to consider the limit $\ell_{\mathrm{P}} \mapsto 0, j \mapsto \infty$ such that $\ell_{\mathrm{P}}^{2} j$ is constant, to study the semiclassical behavior of the 2-point function. This idea is supported by the asymptotic behavior of the $\{10 j\}$ symbol for large spins, which we recall here.

In the homogeneous large spin limit, namely when $j_{l}=N k_{l}$ and $N \mapsto \infty$, the integral (66) defining the $\{10 j\}$ symbol can be evaluated with a saddle point approximation, leading to the asymptotics [11, 12]

$$
\{10 j\} \sim \sum_{\tau} P(\tau) \cos \left(S_{\mathrm{R}}\left[j_{l}\right]+\kappa_{\tau} \frac{\pi}{4}\right)+D\left(j_{l}\right)
$$

Here $S_{\mathrm{R}}\left[j_{l}\right]=\sum_{l} d_{j_{l}} \theta_{l}$ is the Regge action associated with the 4-simplex, dual to the foam vertex, with triangle areas $A_{l}=d_{j_{l}} ; P(\tau)$ and $\kappa_{\tau}$ are factors depending on the combinatorial structure of the 4-simplex, and $D\left(j_{l}\right)$ is a contribution coming from degenerate configurations of the 4-simplex. In principle, the emergence of the Regge action

2 In the literature, the edge (or tetrahedron) amplitude $A_{e}\left(j_{1}, j_{2}, j_{3}, j_{4}\right)$ is usually the product of some particular powers of $d_{j_{1}} d_{j_{2}} d_{j_{3}} d_{j_{4}}$ and $d_{j_{1} j_{2} j_{3} j_{4}}$, where the latter is the dimension of the intertwiner space and is given by the norm of the Barrett-Crane intertwiner:

$$
d_{j_{1} j_{2} j_{3} j_{4}}=\int_{\mathrm{SU}(2)} d g \prod_{i=1}^{4} \chi_{j_{i}}(g) .
$$


supports the idea that the large $j$ limit can be used to study the semiclassical properties of the theory. However, the degenerate term $D\left(j_{l}\right)$ dominates strongly the asymptotics, thus hiding the physically interesting first term of (14). Indeed, the numerical analysis in [13] could not confirm (14). This fact has raised doubts over the BC model. Nevertheless, it was suggested in [1] that the sick term $D\left(j_{l}\right)$ is in fact negligible in the computation of (11), because it does not match the boundary data induced by $\Psi_{0}\left[j_{l}\right]$. Before showing how this happens, let us discuss the structure of the perturbative expansion.

Using the approximation (14) in (11), we compute the 2-point function within a particular Regge path integral formulation of quantum gravity. The measure for this path integral is induced by the spinfoam formalism. On the other hand, the Regge action is a discretised version of GR, and it can be expanded around the flat background, as in the continuum. The quadratic term in the action is responsible for the behavior of the leading order of the 2 -point function. Higher order terms in the action give corrections in powers of $\ell_{\mathrm{P}}$. However, because (14) is only an approximation, we expect to have higher order corrections to the 2-point function which are not described by Regge calculus. Indeed, this expectation is confirmed in $3 \mathrm{~d}[4]$, where an interesting structure of the corrections emerges.

This is not the end of the story. For any $s$ in (11), the amplitude (13) sums over all spin foams interpolating $s$. Using as in [1] the group field theory generated BC model, each foam is weighted by a factor $\lambda^{V}$, where $V$ is the total number of vertices in $\sigma$, and $\lambda$ is the dimensionless coupling constant of the group field theory. Assuming that the perturbative expansion in $\lambda$ is well defined, this suggests that from the group field theory point of view, the dominant contributions come from the simplest foams. Indeed, at first order in $\lambda$ we have a single contribution to (11) (see [5]), whose boundary is the 4-simplex spin network.

Understanding the precise interplay between the $\ell_{\mathrm{P}}$ expansion and the $\lambda$ expansion is beyond the scope of this paper. In the rest of this paper, we focus on this single contribution. See the conclusions for more comments.

\section{The Gaussian boundary state and the leading order of the perturbative expansion}

Let us recall here the contribution to the 2-point function coming from a single 4-simplex, as computed in [1]. When we restrict the boundary $s$ to be only the pentahedral spin network, (11) reduces to

$$
W_{a b}\left(j_{0}\right)=\frac{1}{j_{0}^{4}} \frac{1}{\mathcal{N}} \sum_{j_{l}} \mathcal{A}\left[j_{l}\right] \Psi_{0}\left[j_{l}\right] \mathbb{h}\left(j_{a}\right) \mathfrak{h}\left(j_{b}\right) .
$$

To explicitly define $\mathcal{A}\left[j_{l}\right]$, we need to make a choice for the edge amplitudes $A_{e}$. The amplitude $\mathcal{A}\left[j_{l}\right]$ will then involve the face factors $d_{j}^{2}$ and the square-root of the boundary edge amplitudes $\left(A_{e}\right)^{1 / 2}$. We choose $A_{e}\left(j_{f}\right)=\left(\prod_{f} d_{j_{f}}\right)^{-1}$. Taking into account the five tetrahedra of the boundary of the 4-simplex, we have $\prod_{e \in \partial \sigma} \sqrt{A_{e}\left(j_{f}\right)}=\prod_{f} \prod_{e} d_{j_{f}}^{-1 / 2} \equiv$ $\prod_{f} d_{j_{f}}^{-1}$ and thus:

$$
\mathcal{A}\left[j_{l}\right]=\prod_{l} d_{j_{l}}^{2} \prod_{l} \frac{1}{d_{j_{l}}}\{10 j\}=\prod_{l} d_{j_{l}}\{10 j\}=\int \prod_{n} d g_{n} \prod_{l} \chi_{j_{l}}\left(g_{s(l)}^{-1} g_{t(l)}\right) .
$$

To compute the free propagator, namely the leading order in the large $j_{0}$ expansion of (11), it is sufficient to consider a Gaussian state (see discussion in [5]) peaking the (discrete variables representing the) boundary geometry (encoded in the spin network labels $j_{l}$ ) around a given semiclassical configuration. Choosing the equilateral configuration as the background geometry of the boundary 4 -simplex, the data encoded by $\Psi_{0}\left[j_{l}\right]$ are the value $2 j_{0}$ of the area of the ten triangles, representing the intrinsic curvature, and the value $\theta=\arccos \left(-\frac{1}{4}\right)$ of the ten dihedral angles, representing the extrinsic curvature. In particular in [1], the following ansatz was taken:

$$
\Psi_{0}\left[j_{l}\right]=\exp \left\{-\frac{1}{2 j_{0}} \sum_{l l^{\prime}} \alpha_{l l^{\prime}} \delta j_{l} \delta j_{l^{\prime}}+i \theta \sum_{l}\left(2 j_{l}+1\right)\right\},
$$

where $\delta j_{l}=j_{l}-j_{0}$, and $\alpha_{l l^{\prime}}$ is a $\mathbb{C}$-valued (non diagonal) matrix that can be fixed by comparing the leading order of (11) with (a suitable discretisation of) the conventional free propagator [1]. Using (17), we have $\frac{\left\langle\Delta j_{l}\right\rangle}{\left\langle j_{l}\right\rangle}=\frac{1}{\sqrt{j_{0} \alpha_{l l}}}$ and $\frac{\left\langle\Delta \phi_{l}\right\rangle}{\left\langle\phi_{l}\right\rangle}=\frac{1}{\theta} \sqrt{\frac{\alpha_{l l}}{j_{0}}}$, so that (12) is satisfied in the $j_{0} \mapsto \infty$ limit. 
It was assumed that the phase of (17) suppresses the term $D\left(j_{l}\right)$. Then, using (14) and (17), in [1] it was showed that the leading order of (15), in the large spin limit, is

$$
W \sim \frac{1}{j_{0}} .
$$

This shows that the leading order of the components of the 2-point function behave as expected: rescaling the boundary geometry where the two points lie, $W_{a b}$ scales as $1 /|x-y|^{2}$.

However this evaluation relies on a number of assumptions. In particular the use of (14), and the suppression of the degenerate configurations term $D\left(j_{l}\right)$. It would thus be important to confirm the analytic result (18) with numerical simulations of (15), but this has not been achieved so far [14, due to the high complexity of the sum. In the rest of this paper we address this issue, and we prove (18) in a way that has been verified numerically [14]. In particular, we do not use (14), and we prove that the degenerate configurations plaguing the BC model are indeed suppressed. First of all, notice that using the integral representation (16) of the spinfoam amplitude, and swapping the integrals with the sums, we can rewrite (15) as

$$
W_{a b}=\frac{1}{j_{0}^{4}} \frac{1}{\mathcal{N}} \int \prod_{n} d g_{n} \sum_{j_{l}} \prod_{l} \chi_{j_{l}}\left(g_{s(l)}^{-1} g_{t(l)}\right) \Psi_{0}\left[j_{l}\right] \mathbb{h}\left(j_{a}\right) \mathbb{h}\left(j_{b}\right) .
$$

The key idea is to use a boundary state $\Psi_{0}\left[j_{l}\right]$, so that we can perform exactly the sums in (29). To do so, recall that the kernel is nothing but the $\mathrm{SU}(2)$ character. Then, to be able to perform the sum, it is sufficient to have a state $\Psi_{0}\left[j_{l}\right]$ with a well-defined Fourier transform. This is what we do in the next Section.

\section{THE NEW BOUNDARY STATE}

As we showed above the relevant group for 4d Riemannian GR without matter is simply SU(2); indeed, (11) uses only SU(2) spins. This has interesting consequences, as $\mathrm{SU}(2)$ is also the relevant group for 3d Riemannian GR. Therefore we can apply to the Barrett-Crane model in 4d the same techniques developed to study the graviton propagator in the Ponzano-Regge model for 3d quantum gravity in [4]. In particular, we consider the following new ansatz for the boundary state,

$$
\begin{aligned}
& \Psi_{0}\left(j_{l}\right)=\prod_{l} \psi_{0}\left(j_{l}\right) \\
& \psi_{0}(j)=\frac{I_{\left|j-j_{0}\right|}\left(\frac{j_{0}}{\alpha}\right)-I_{j+j_{0}+1}\left(\frac{j_{0}}{\alpha}\right)}{\sqrt{I_{0}\left(\frac{2 j_{0}}{\alpha}\right)-I_{2 j_{0}+1}\left(\frac{2 j_{0}}{\alpha}\right)}} \cos \left(d_{j} \theta\right) .
\end{aligned}
$$

Here the $I_{n}(z)$ are the modified Bessel functions of the first kind, and $\alpha \in \mathbb{R}^{+}$is a free parameter. ${ }^{3}$

Notice that this new boundary state factorises in link contributions and is real, differently from (17). As shown in [4], the $j_{0} \mapsto \infty$ limit of (21) behaves as a Gaussian peaked around $j_{0}$,

$$
\psi_{0}(j) \simeq \sqrt[4]{\frac{\alpha}{j_{0} \pi}} \exp \left\{-\frac{\alpha}{2 j_{0}}\left(j-j_{0}\right)^{2}\right\} \cos \left(d_{j} \theta\right)
$$

and thus (20) satisfies the semiclassical requirements (12). Thanks to the asymptotic behavior (22), a boundary state constructed from (21) can be used to reproduce the leading order (18) using the same procedure outlined in the previous Section.

\footnotetext{
${ }^{3}$ With respect to [4], there is an important difference in the phase, which is given by $d_{j} \theta$ here and by $d_{j} \theta / 2$ in the $3 \mathrm{~d}$ case. The reason for this lies in the asymptotics [14], which reproduce the Regge action with areas $A=d_{j}$, whereas the asymptotics for the $3 \mathrm{~d}$ spinfoam amplitude reproduce the Regge action with lengths $\ell=d_{j} / 2$.
} 
However, the exact form (21) opens the way to a new treatment, which allows us give a check of the procedure of [1]. The key property of (21) which we need in the following is its Fourier transform (see [4]),

$$
\widetilde{\psi}_{0}(g)=\sum_{j} \psi_{0}(j) \chi_{j}(g)=\sum_{\eta= \pm} \frac{e^{-\frac{2 j_{0}}{\alpha} \sin ^{2}(\phi-\eta \theta)}}{2 N \sin \phi} \sin \left(d_{j_{0}}(\phi-\eta \theta)\right) .
$$

where

$$
N=e^{-\frac{j_{0}}{\alpha}} \sqrt{I_{0}\left(\frac{2 j_{0}}{\alpha}\right)-I_{2 j_{0}+1}\left(\frac{2 j_{0}}{\alpha}\right)}
$$

is the normalisation with respect to the Haar measure. From the Gaussian shape of (23), it is clear that this new boundary state peaks the $\mathrm{SU}(2)$ class angle $\phi$ around the values $\eta \theta$ modulus $\pi$. For later purpose, we introduce a sign variable $\sigma= \pm$ and write the peaks as

$$
\bar{\phi}=\eta \theta+\frac{1-\sigma}{2} \pi .
$$

As we show below, the different values of the signs $\eta$ and $\sigma$ give the same contribution to the 2-point function, thus summing over them simply adds a trivial redundancy.

The fact that (21) admits a simple Fourier transform is at the heart of our construction of the 2-point function as an integral over $\mathrm{SU}(2)$. In particular, in the following we also need the convolution product between $\psi_{0}$ and the field insertion $\mathfrak{h}, \psi_{0} \circ \mathbb{h}$. This can be easily evaluated, using

$$
\nabla^{2}=\frac{1}{\sin ^{2} \phi} \partial_{\phi} \sin ^{2} \phi \partial_{\phi}, \quad \nabla^{2} \chi_{j}(\phi)=-4 j(j+1) \chi_{j}(\phi),
$$

to write

$$
\begin{aligned}
& \left(\psi_{0} \circ \mathbb{h}\right)(\phi)=\sum_{j} \chi_{j}(\phi) \Psi_{0}(j)\left[C^{2}(j)-C^{2}\left(j_{0}\right)\right]=\left[-\frac{1}{4} \nabla^{2}-j_{0}\left(j_{0}+1\right)\right] \widetilde{\psi}_{0}(\phi)= \\
& =\sum_{\eta= \pm} \frac{j_{0}}{\alpha} \frac{e^{-\frac{2 j_{0}}{\alpha} \sin ^{2}(\phi-\eta \theta)}}{2 N \sin \phi} \sin \left(d_{j_{0}}(\phi-\eta \theta)\right) \sin 2(\phi-\eta \theta)\left[\cot 2(\phi-\eta \theta)-\frac{j_{0}}{\alpha} \sin 2(\phi-\eta \theta)+d_{j_{0}} \cot \left(d_{j_{0}}(\phi-\eta \theta)\right)\right] .
\end{aligned}
$$

For later use, let us rewrite this expression as

$$
\left(\psi_{0} \circ \mathbb{h}\right)(\phi)=\sum_{\epsilon= \pm} \sum_{\eta= \pm} \frac{j_{0}}{\alpha} \frac{e^{-\frac{2 j_{0}}{\alpha} \sin ^{2}(\phi-\eta \theta)}}{2 N \sin \phi} \epsilon e^{i \epsilon d_{j_{0}}(\phi-\eta \theta)} \sin 2(\phi-\eta \theta)\left[\cot 2(\phi-\eta \theta)-\frac{j_{0}}{\alpha} \sin 2(\phi-\eta \theta)+d_{j_{0}} i \epsilon\right] .
$$

Before proceeding, let us add a few remarks on the new boundary state, which make it particularly appealing.

- The spin and the angle entering the state, which represent a discretised version of the conjugate intrinsic and extrinsic curvature variables, are conjugate variables in a precise mathematical sense: they are conjugate with respect to the $\mathrm{SU}(2)$ harmonic analysis.

- The state is a real quantity. This is due to the fact that the phase is given by a cosine, and not a single exponent, as in (17). The fact that using the cosine and not just a single exponent does not spoil the leading order of (11) was proved in [4]. Furthermore, one could also consider a sine term, or equivalently an SU(2) character. In 3d, this can be related to particle insertions [4, 16].

- The boundary state has an interacting structure between the variables and the background; it reduces to a Gaussian only in the limit $j_{0} \mapsto \infty$. For this reason it will also contribute to the corrections to the free propagator. In particular, the $3 \mathrm{~d}$ analysis of this state shows that its contribution interestingly reduces the magnitude of the next to leading order. 
- The boundary state (20) is factorised into contributions from single links of the boundary spin network. This makes its analysis much simpler than (17), but also means that it is straightforward to extend it to arbitrary triangulations.

- In the definition (20) we took the same $\alpha$ for all links. Nothing prevents us from considering arbitrary configurations with a different parameter for each link. Because this parameter is in a sense related to the knowledge of the boundary geometry, taking the same value for all links amounts to a homogeneous description of the boundary state.

\section{THE 2-POINT FUNCTION AS AN INTEGRAL OVER SU(2)}

Thanks to the factorisation of the new boundary state, we can rewrite (19) as

$$
W_{a b}=\frac{1}{j_{0}^{4}} \frac{1}{\mathcal{N}} \int \prod_{n} d g_{n} \prod_{l} \mathcal{I}_{l}\left(g_{l}\right)
$$

where $g_{l}=g_{s(l)}^{-1} g_{t(l)}$, and

$$
\mathcal{I}_{l}(g)=\left\{\begin{array}{l}
\sum_{j_{l}} \chi_{j_{l}}(g) \psi_{0}\left[j_{l}\right] \equiv \widetilde{\psi}_{0}(g), \quad \text { if } \quad l \neq a, b, \\
\sum_{j_{l}} \chi_{j_{l}}\left(g_{l}\right) \psi_{0}\left[j_{l}\right] \mathfrak{h}\left(j_{l}\right) \equiv\left(\psi_{0} \circ \mathfrak{h}\right)(g) \quad \text { if } \quad l=a, b,
\end{array}\right.
$$

are given respectively by (23) and (27). This expression gives the graviton propagator as an integral over SU(2).

The choice of the adge amplitude $A_{e}\left(j_{f}\right)=\left(\prod_{f} d_{j_{f}}\right)^{-1}$ made above simplifies the computation of the two Fourier transforms. More generically, if $A_{e}\left(j_{f}\right)$ does not couple the spins and is simply a power of $\prod_{f} d_{j_{f}}, W_{a b}$ still factorises as above, with the extra powers of $d_{j_{l}}$ acting as differential operators $\partial_{\theta}$ in computing the Fourier transform. On the other hand, if the edge amplitude couples the spins and introduces some $d_{j_{1} j_{2} j_{3} j_{4}}$ factors (see footnote 2), the situation is different. For a single 4-simplex, these factors can always be compensated by introducing suitable counter-factors in the boundary state, and thus the form (29) can be restored. For multi-simplices configurations, this will not work. However, for arbitrary triangulations it is not straightforward to obtain the integral representation (29) of the graviton, even with the simplest choice $A_{e}\left(j_{f}\right)=\left(\prod_{f} d_{j_{f}}\right)^{-1}$. In fact, the one-to-one correspondence between $\mathrm{SU}(2)$ characters and factors (21) of the boundary state is in general lost. This makes it harder to perform the sums explicitly. We postpone the study of arbitrary triangulations to future work, nonetheless let us notice here that the correspondence is preserved by any $n$-valent vertex, as long as the vertex amplitude is provided by the relativistic spin network (5). Therefore, an integral expression like (29) can be obtained for any triangulation that can be coarse grained to a single $n$-valent vertex.

To explicitly evaluate the perturbative expansion in $j_{0}$, it is convenient to use the measure (8) in terms of the ten angles. Notice that the factors $\sin \phi_{l}$ in (8) simplify with the ones coming from $\tilde{\psi}_{0}(g)$ and $\left(\psi_{0} \circ \mathbb{h}\right)(g)$, and we can write (29) as

$$
W_{a b}=\frac{1}{j_{0}^{4}} \frac{1}{\mathcal{N}} \sum_{\epsilon_{l}= \pm} \sum_{\eta_{l}= \pm} \int \prod_{l} d \phi_{l} \delta\left(\operatorname{det} G_{I J}\right) \prod_{l} \epsilon_{l} e^{i \epsilon_{l} d_{j_{0}}\left(\phi_{l}-\eta_{l} \theta\right)} \mathfrak{h}\left(\phi_{a}\right) \mathfrak{h}\left(\phi_{b}\right) e^{-\frac{2 j_{0}}{\alpha} \sum_{l} \sin ^{2}\left(\phi_{l}-\eta_{l} \theta\right)}
$$

where we have absorbed the constant $(2 N)^{10}$ in the normalisation, and we have introduced the notation

$$
\mathbb{h}\left(\phi_{a}\right)=\frac{j_{0}}{\alpha} \sin 2\left(\phi_{a}-\eta_{a} \theta\right)\left[\cot 2\left(\phi_{a}-\eta_{a} \theta\right)-\frac{j_{0}}{\alpha} \sin 2\left(\phi_{a}-\eta_{a} \theta\right)+d_{j_{0}} i \epsilon_{a}\right] .
$$

The expression (30) is the contribution to the graviton propagator coming from a single 4-simplex.

Let us stress that (30) is an integral over SU(2) with no sums involved, as opposed to (15). This result is particularly important from a numerical point of view. The integral is in fact much easier to handle numerically than the sums, and the formulation provided here gives substantial progress in the numerical simulations [14]. As far as the sum over the $\eta$ signs are concerned, we show below that each configuration gives the same contribution, so that the sum gives a trivial redundancy that can be reabsorbed in the normalisation. 
To define the perturbative expansion in $\ell_{\mathrm{P}}$ we can use the parameter $j_{0}$ as above. Notice that $j_{0}$ enters the exponential of the integrand. Therefore the leading order in the $j_{0} \mapsto \infty$ limit can be computed using the saddle point approximation of the integral. We do so in the next Section.

\section{THE SADDLE POINT APPROXIMATION}

The study of the leading order and corrections to the 2-point function formulated as a group integral requires the analysis the saddle point (or stationary phase) approximation of the following type of integral:

$$
\int d \mu\left[\phi_{l}\right] F\left(\phi_{l}\right) \sum_{\eta_{l}= \pm} e^{i \sum_{l} \epsilon_{l} d_{j_{0}}\left(\phi_{l}-\eta_{l} \theta\right)} e^{-\frac{2 j_{0}}{\alpha} \sum_{l} \sin ^{2}\left(\phi_{l}-\eta_{l} \theta\right)},
$$

where $\epsilon_{l}= \pm$ are signs, $F\left(\phi_{l}\right)$ an arbitrary function (with no dependence on $j_{0}$ ) and the measure $d \mu\left(\phi_{l}\right)$ is defined in (8). For large $j_{0}$ that integral is dominated at the fixed points of the action

$$
S\left[\phi_{l}, \kappa\right]=\sum_{l}\left[\frac{2}{\alpha} \sin ^{2}\left(\phi_{l}-\eta_{l} \theta\right)-2 i \epsilon_{l}\left(\phi_{l}-\eta_{l} \theta\right)\right]-i \frac{\kappa}{j_{0}} \operatorname{det} G_{I J}
$$

where we used a Lagrange multiplier $\kappa$ to introduce the constraint $\operatorname{det} G_{I J}=0$. To compute the equations of motion, notice that $\frac{\partial \operatorname{det} G_{I J}}{\partial \phi_{l}}=-2 \sin \phi_{l} \Lambda^{(l)}$, where $\Lambda^{(l)}$ is the determinant of the off-diagonal $l$-th minor of $G_{I J}$, obtained removing the line and column corresponding to one of the two $\cos \phi_{l}$ appearing in it.

Then the equations of motion read:

$$
\left\{\begin{array}{l}
\frac{2}{\alpha} \sin 2\left(\phi_{l}-\eta_{l} \theta\right)-2 i \epsilon_{l}=-2 i \frac{\kappa}{j_{0}} \sin \phi_{l} \Lambda^{(l)} \quad \forall l \\
\operatorname{det} G_{I J}=0 .
\end{array}\right.
$$

The only real solution to this set of equations is:

$$
\overline{\phi_{l}}=\eta_{l} \theta+\frac{1-\sigma_{l}}{2} \pi, \quad \overline{\epsilon_{l}}=\epsilon \eta_{l}
$$

where $\epsilon= \pm$ is a global sign (independent of the link $l$ ), and it requires the symmetry property $\sigma_{l}=\sigma_{I J}=\sigma_{I} \sigma_{J}$, with $\sigma_{I}$ and $\sigma_{J}$ independent signs. We have $\sin \overline{\phi_{l}}=\eta_{l} \sigma_{l} \sin \theta$ and $\Lambda^{(l)}\left(\overline{\phi_{l}}\right)=\sigma_{l} \Lambda_{0}, \Lambda_{0}:=\cos \theta(1-\cos \theta)^{3}$. Consequently, the Lagrange multiplier takes the value

$$
\bar{\kappa}=\epsilon \frac{j_{0}}{\sin \theta \Lambda_{0}} .
$$

The action evaluated on the fixed point (34) is purely real and vanishes, $S\left[\overline{\phi_{l}}, \bar{\kappa}\right]=0$. If $\epsilon_{l} \neq \overline{\epsilon_{l}}$, there is no real solution. The saddle point approximation selects two specific terms out of the sum over all possible sign assignments.

More generally, the first part of the action, $S_{0}[\phi, \kappa]=-\sum_{l} 2 i \epsilon_{l} \phi_{l}-i \frac{\kappa}{j_{0}} \operatorname{det} G_{I J}$, is the standard action governing the asymptotics of the (equilateral) $\{10 j\}$ symbol. It has two non-degenerate fixed points $\phi_{l}=\theta$ and $\phi_{l}=-\theta$, but also degenerate fixed points at $\phi_{l}=0, \pi$. These degenerate fixed points dominate the asymptotic behavior of the $\{10 j\}$ symbol [11, 12, 13], and are responsible for the $D$ term in (14). However, we see that their contribution to the 2-point function is suppressed: in (30), there is an additional Gaussian weight, which is maximized by $\phi_{l}=\theta$ but not by $\phi=0, \pi$. Therefore it kills the degenerate configurations and peaks the asymptotics around the non-degenerate semiclassical configuration. This provides the mechanism to neglect the $D$ term, as done in [1].

We have not shown that there is no complex solution to these equations although we believe there is none. ${ }^{4}$ In particular, there could be fixed points close or along the imaginary line $\phi_{l}-\eta_{l} \theta \in i \mathbb{R}$, for which the Gaussian would

\footnotetext{
${ }^{4}$ At least, it is fairly straightforward to check that there is no purely imaginary solutions $\phi_{l} \in i \mathbb{R}$.
} 
blow up. Such points can be easily found if $\operatorname{det} G_{I J} \neq 0$ is allowed, but otherwise we have not been able to find any. The results of our partial analysis suggest that the constraint $\operatorname{det} G_{I J}=0$ protects the integral from the presence of exponentially enhanced fixed points, a mechanism that could be relevant also for the asymptotics of the $\{10 j\}$ symbol alone.

Before proceeding, let us add an important remark. ${ }^{5}$ After the identification of the fixed points, one usually computes the integral along the complex contour such that the phase (namely the imaginary part of $S$ ) varies the least. This steepest descent method is implemented imposing that the imaginary part of the second derivative of $S$ vanishes. However, in the following we will use the real contour, which does not satisfy this requirement (as can be immediately seen from the explicit expression of the second derivatives in (33)). Nevertheless, as we mention at the end of this section, a numerical check has been performed independently from our analysis and the fit is (at least) of two decimals. This means that most likely there are no other (relevant) fixed points and that the real contour is enough for the study of the correlations.

The fact that complex fixed points, if they do exist, are not relevant for our analysis may be due to the following reasons. First, notice that we are summing over all signs $\eta_{l}$, in such a way that only the real part of the integral matters (see below), a fact that might lessen the relevance of the phase. Then, recall that the precise normalisation of the integral is not relevant but only its ratio with $\mathcal{N}$, the integral without the $\mathbb{h}$ insertions. Finally, we are merely computing the first order of the large $j_{0}$ asymptotics of $W_{a b}$. The existence of complex fixed points might become relevant when computing the higher order corrections. We leave this question open for future investigations.

Based on this preliminary analysis of the stationary points, we are now ready to state the main result of this paper on the asymptotics of the 4-simplex 2-point function.

Theorem 1. The leading order of the large $j_{0}$ expansion of (30) is

$$
W_{a b}\left(j_{0}\right)=\frac{f_{a b}(\alpha, \theta)}{j_{0}},
$$

where $f_{a b}$ has only three independent entries.

These correspond to the three independent projections of the graviton propagator along couples of normals belonging to the same triangles (see discussion in Section III), and should not be confused with the physical independent components. The explicit structure of $f_{a b}(\alpha)$, reported in the proof below, is rather complicate, reflecting the complicate geometrical structure of a 4-simplex, even in the equilateral case.

Proof. The proof goes as follows.

1. We rewrite (30) introducing a Lagrange multiplier $\kappa$ for the constraint $\operatorname{det} G_{I J}$,

$$
W_{a b}=\frac{1}{j_{0}^{4}} \frac{1}{\mathcal{N}} \sum_{\eta_{l}= \pm} \sum_{\epsilon_{l}= \pm} \int d \kappa \int \prod_{l} d \phi_{l}\left(\prod_{l} \epsilon_{l}\right) e^{-j_{0} S\left[\phi_{l}, \kappa\right]+i \sum_{l} \epsilon_{l}\left(\phi_{l}-\eta_{l} \theta\right)} \mathbb{h}\left(\phi_{a}\right) \mathbb{h}\left(\phi_{b}\right),
$$

where $S\left[\phi_{l}, \kappa\right]$ is given by (32). The normalisation $\mathcal{N}$ is given by the same quantity above without the insertions $\mathbb{h}\left(\phi_{a}\right) \mathfrak{h}\left(\phi_{b}\right)$ and without the constant factor $j_{0}^{-4}$. In the calculations below, we will use $\mathcal{N}$ to reabsorb a number of overall constants, without affecting the final result.

The expansion parameter $j_{0}$ enters the exponent in front of the action $S$, and the field insertions $\mathbb{h}$. The saddle point of the action is

$$
\phi_{l}=\overline{\phi_{l}}, \quad \epsilon_{l}=\overline{\epsilon_{l}}, \quad \kappa=\bar{\kappa},
$$

where $\overline{\phi_{l}}$ and $\overline{\epsilon_{l}}$ are given in (34), and $\bar{\kappa}$ in (35).

\footnotetext{
${ }^{5}$ We thank our referee for pointing this out.
} 
2. To compute the leading order of (37), we expand the action to second order around the saddle point (where both the zeroth and the linear orders vanish), and evaluate the rest at lowest order. In particular, at the saddle point we have

$$
\left.\prod_{l} \epsilon_{l}\right|_{\mathrm{saddle}}=\prod_{l} \epsilon \eta_{l} \equiv \prod_{l} \eta_{l},\left.\quad e^{i \sum_{l} \epsilon_{l}\left(\phi_{l}-\eta_{l} \theta\right)}\right|_{\mathrm{saddle}}=\prod_{l} e^{i \epsilon \eta_{l} \frac{1-\sigma_{l}}{2} \pi}=\prod_{l} \sigma_{l} .
$$

Consequently, we obtain the global sign $\prod_{l} \eta_{l} \sigma_{l}$. When we perform the sum over the $\eta_{l}$ signs in (37), we obtain identically zero unless we have the matching $\eta_{l}=\sigma \sigma_{l} \forall l$, where $\sigma$ is an irrelevant overall sign. In the following, we take $\sigma_{l}=\eta_{l}$.

The leading order of (37) is thus

$$
W_{a b} \simeq \frac{1}{j_{0}^{4}} \frac{1}{\mathcal{N}} \sum_{\eta_{l}= \pm} \sum_{\epsilon= \pm} \int d \delta \kappa \int \prod_{l} d \delta \phi_{l} e^{-\frac{j_{0}}{2}\left(\frac{\partial^{2} S}{\partial \phi_{l} \partial \phi_{l^{\prime}}} \delta \phi_{l} \delta \phi_{l^{\prime}}+\frac{\partial^{2} S}{\partial \phi_{l} \partial \kappa} \delta \phi_{l} \delta \kappa\right)} \mathfrak{h}\left(\overline{\phi_{a}}+\delta \phi_{a}\right) \mathbb{h}\left(\overline{\phi_{b}}+\delta \phi_{b}\right) .
$$

3. In principle, the value of the field insertions at the saddle point is enough for the leading order. However, we have $\mathbb{h}(\bar{\phi}) \equiv 0$, thus we need to expand the field insertions. This can be done in two steps. First, we keep only the terms in $j_{0}^{2}$ in (31), thus

$$
\mathbb{h}\left(\phi_{a}\right) \simeq\left(\frac{j_{0}}{\alpha}\right)^{2} \sin 2\left(\phi_{a}-\eta_{a} \theta\right)\left[2 \alpha i \epsilon_{a}-\sin 2\left(\phi_{a}-\eta_{a} \theta\right)\right] .
$$

Then, we expand $\phi=\bar{\phi}+\delta \phi$. We have $\sin \left(\left(1-\sigma_{a}\right) \pi+2 \delta \phi_{a}\right)=\sin 2 \delta \phi_{a} \simeq 2 \delta \phi_{a}$. Using also $\epsilon_{a}=\epsilon \eta_{a}$, we can write

$$
\mathrm{h}\left(\bar{\phi}_{a}+\delta \phi_{a}\right) \simeq\left(\frac{j_{0}}{\alpha}\right)^{2} 2 \delta \phi_{a}\left[2 \alpha i \epsilon \eta_{a}-2 \delta \phi_{a}\right] .
$$

However, these two terms are of different orders. In fact, notice that (40) is a Gaussian integral with width proportional to $1 / \sqrt{j_{0}}$. Therefore $\delta \phi^{2} \sim 1 / j_{0}$, so the second term in (42) can be neglected, and finally

$$
\mathbb{h}\left(\bar{\phi}_{a}+\delta \phi_{a}\right) \simeq 4 \alpha i\left(\frac{j_{0}}{\alpha}\right)^{2} \epsilon \eta_{a} \delta \phi_{a} .
$$

4. The second derivatives of the action have the following form,

$$
\left.\frac{\partial^{2} S}{\partial \phi_{l} \partial \phi_{l^{\prime}}}\right|_{\text {saddle }}=\eta_{l} \eta_{l^{\prime}} A_{l l^{\prime}}^{\epsilon},\left.\quad \frac{\partial^{2} S}{\partial \phi_{l} \partial \kappa}\right|_{\text {saddle }}=\frac{2 i}{j_{0}} \eta_{l} \sin \theta \Lambda_{0} .
$$

The explicit form of $A_{l l^{\prime}}^{\epsilon}$ is given in the Appendix, and it satisfies $A_{l l^{\prime}}^{-}=\overline{A_{l l^{\prime}}^{+}}$.

Notice that the constant factor $\sin \theta \Lambda_{0}$ can be neglected rescaling the definition of $\delta \kappa$, and then reabsorbing it in the normalisation $\mathcal{N}$ of (40).

5. The leading order of (37) is thus the following Gaussian integral,

$$
\begin{aligned}
W_{a b} & =-\frac{1}{\mathcal{N}} \frac{16}{\alpha^{2}} \sum_{\eta_{l}= \pm} \sum_{\epsilon= \pm} \eta_{a} \eta_{b} \int d \delta \kappa \int \prod_{l} d \delta \phi_{l} \delta \phi_{a} \delta \phi_{b} \exp \left[-\eta_{l} \eta_{l^{\prime}} \frac{j_{0}}{2} A_{l l^{\prime}}^{\epsilon} \delta \phi_{l} \delta \phi_{l^{\prime}}-i \eta_{l} \delta \phi_{l} \delta \kappa\right]= \\
& =-\left.\frac{16}{\alpha^{2}} \frac{1}{Z(0)} \frac{\partial^{2}}{\partial J_{a} \partial J_{b}} Z(J)\right|_{J=0},
\end{aligned}
$$

where we have introduced the generating functional

$$
Z(J)=\sum_{\eta_{l}= \pm} \sum_{\epsilon= \pm} \int d \delta \kappa \int \prod_{l} d \delta \phi_{l} \exp \left[-\eta_{l} \eta_{l^{\prime}} \frac{j_{0}}{2} A_{l l^{\prime}}^{\epsilon} \delta \phi_{l} \delta \phi_{l^{\prime}}-i \eta_{l} \delta \phi_{l}\left(\delta \kappa+i J_{l}\right)\right] .
$$


6. The generating functional is a Gaussian integral that can be evaluated straightforwardly. To maintain the explicit symmetry of the problem, we perform first the integral over the ten angles, obtaining

$$
Z(J)=\sum_{\eta_{l}= \pm} \sum_{\epsilon= \pm} \frac{(2 \pi)^{5}}{j_{0}^{5} \sqrt{\operatorname{det} A_{l l^{\prime}}^{\epsilon}}} \int d \delta \kappa \exp \left[-\frac{1}{2 j_{0}} \sum_{l l^{\prime}}\left(\delta \kappa+i J_{l}\right)\left(A_{\epsilon}{ }^{-1}\right)_{l l^{\prime}}\left(\delta \kappa+i J_{l^{\prime}}\right)\right]
$$

Here we used the fact that $\operatorname{det}\left(\eta_{l} \eta_{l^{\prime}} A_{l l^{\prime}}^{\epsilon}\right) \equiv \operatorname{det} A_{l l^{\prime}}^{\epsilon}$. Observe that consequently the sums over the $\eta_{l}$ signs give a trivial redundancy $2^{10}$. The remaining integral is also straightforward, and we finally obtain

$$
Z(J)=2^{10} \sum_{\epsilon= \pm} \frac{(2 \pi)^{5} \sqrt{2 \pi j_{0}}}{j_{0}^{5} \sqrt{\operatorname{det} A_{l l^{\prime}}^{\epsilon} \sum_{l l^{\prime}}\left(A_{\epsilon}^{-1}\right)_{l l^{\prime}}}} \exp \left[\frac{1}{2 j_{0}}\left(\sum_{l l^{\prime}}\left(A_{\epsilon}{ }^{-1}\right)_{l l^{\prime}} J_{l} J_{l^{\prime}}-\frac{\left(\sum_{l l^{\prime}}\left(A_{\epsilon}^{-1}\right)_{l l^{\prime}} J_{l}\right)^{2}}{\sum_{l l^{\prime}}\left(A_{\epsilon}^{-1}\right)_{l l^{\prime}}}\right]\right.
$$

Because $A_{l l^{\prime}}^{-}=\overline{A_{l l^{\prime}}^{+}}$, the sum over the $\epsilon$ sign amounts to taking (twice) the real part of the summand. Reabsorbing the irrelevant constants in the normalisation $Z(0)$ and defining $A_{l l^{\prime}}=A_{l l^{\prime}}^{+}$, we can then write

$$
Z(J)=\operatorname{Re}\left\{\frac{1}{\sqrt{\operatorname{det} A_{l l^{\prime}} \sum_{l l^{\prime}}\left(A^{-1}\right)_{l l^{\prime}}}} \exp \left[\frac{1}{2 j_{0}}\left[\sum_{l l^{\prime}}\left(A^{-1}\right)_{l l^{\prime}} J_{l} J_{l^{\prime}}-\frac{\left(\sum_{l l^{\prime}}\left(A^{-1}\right)_{l l^{\prime}} J_{l}\right)^{2}}{\sum_{l l^{\prime}}\left(A^{-1}\right)_{l l^{\prime}}}\right]\right] .\right.
$$

7. As shown in the Appendix, the matrix $\left(A^{-1}\right) l l^{\prime}$ satisfies $\sum_{l^{\prime}}\left(A^{-1}\right) l l^{\prime}=f_{1}(\alpha) \forall l$, where $f_{1}(\alpha)=\frac{\sqrt{15}}{2\left(\frac{2 \sqrt{15}}{\alpha}+5 i\right)}$, and thus $\sum_{l l^{\prime}}\left(A^{-1}\right)_{l l^{\prime}}=10 f_{1}(\alpha)$.

Using this, we can finally write

$$
W_{a b}=-\frac{1}{\mathcal{N}} \frac{16}{\alpha^{2} j_{0}} \operatorname{Re}\left\{\frac{A_{a b}^{-1}-\frac{1}{10} f_{1}(\alpha)}{\sqrt{\operatorname{det} A 10 f_{1}(\alpha)}}\right\}, \quad \mathcal{N}=\operatorname{Re}\left\{\frac{1}{\sqrt{\operatorname{det} A 10 f_{1}(\alpha)}}\right\}
$$

8. The theorem is proved with

$$
f_{a b}(\alpha)=-\frac{16}{\alpha^{2}} \frac{\operatorname{Re}\left\{\frac{A_{a b}^{-1}-\frac{1}{10} f_{1}(\alpha)}{\sqrt{\operatorname{det} A f_{1}(\alpha)}}\right\}}{\operatorname{Re}\left\{\frac{1}{\sqrt{\operatorname{det} A f_{1}(\alpha)}}\right\}}
$$

If seen as a matrix, $f_{a b}(\alpha)$ has only three independent components, as it is shown in the Appendix. Geometrically, these are related to the three cases (i) when $a$ and $b$ are the same triangle, (ii) when they share a side (they are "adjacent" in the 4-simplex), or (iii) when they share only one point (they are "opposite" in the 4-simplex).

For instance, choosing the value $\alpha=1 / 2$, we have

$$
W_{\mathrm{adj}}\left(j_{0}\right) \simeq \frac{1.21}{j_{0}}, \quad W_{\mathrm{opp}}\left(j_{0}\right) \simeq-\frac{0.61}{j_{0}}
$$

Remarkably, these values can be confirmed by a numerical analysis of (37) [14]. 


\section{CONCLUSIONS AND OUTLOOK}

We have introduced a new boundary state to construct the graviton propagator. This new boundary state is given in (20), and in the large spin limit it reduces to the same Gaussian considered in [1]. Using the new state we were able to write the (contribution from a single 4-simplex to the) graviton propagator as an integral over SU(2). This is explicitly given in (30). The result is very useful from the point of view of numerical analysis (see [14]), which is significantly simplified with respect to the expression of [1]. Also, the integral expression of the graviton allows to recast the $\ell_{\mathrm{P}}$ expansion into the saddle point expansion of the integral. Here we evaluated the leading order, given in (36). The reason why the degenerate configurations of the Barrett-Crane model can be neglected emerges clearly: they correspond to non absolute minima of the action. As such, they do not enter the perturbative expansion.

These results provide a starting point for further developments. Let us mention a few which we regard as particularly interesting:

- Compute the next to leading order correction. This is a formidable task, but the integral expression here obtained allows a precise setting to do it. One has to consider higher orders in the expansion of the action (32) around the saddle point, as well as higher orders in the expansion of the field insertions. Furthermore, we expect different choices of edge amplitudes to affect the next to leading order (as it happens in 3d [4]), and thus computing it should allow to discriminate between the different versions of the Barrett-Crane model: different choices of coupling between the 4-simplices will lead to different higher orders. From this point of view, the fact that the expression can also be studied numerically provides a crucial support.

- Compute the angle correlations. This permits to reconstruct the whole tensorial structure of (10), and check the number of physical degrees of freedom. Up to now, we have looked at correlations between the spins $j$ associated to two triangles of the 4 -simplex. These area correlations correspond to components of the type $W_{a a b b}(x, y)$. To access the remaining components, we need to look at observables involving four different triangles of the 4-simplex. This corresponds to correlations between dihedral angles, i.e. between two intertwiners within the 4-simplex. Looking at such correlations was also suggested in [17] in order to study the possibility of long-range correlations in BC-like spinfoam models.

- The boundary state has a parameter, $\alpha$, in some sense related to the knowledge of the boundary geometry. Can we consider also the angle $\theta$ as an external parameter, to be related to the choice of triangulation? This is an interesting question that can be addressed looking at what happens if we choose an angle $\tilde{\theta} \neq \theta=\arccos (-1 / 4)$ in the boundary state (20). The phase of the boundary state would not match anymore the oscillating term of the $\{10 j\}$ symbol and the saddle point approximation would fail. This means that the single 4 -simplex would not give the leading order of the correlation anymore: a different spacetime triangulation is needed to obtain the $1 / j_{0}$ behaviour. For example, for an angle $\tilde{\theta}=n \theta$ we can imagine a configuration with $n 4$-simplices to dominate over the single 4 -simplex configuration, and possibly give the $1 / j_{0}$ behaviour. Notice that the new triangulation giving the leading order would depend on the group field theory coupling constant $\lambda$ and the choice of coupling between 4-simplices (edge amplitude). More work on many 4-simplices configurations, following the original analysis of [5], is needed in order to understand these situations and possibly highlight the precise physical role of $\lambda$.

- Consider the Lorentzian case. Now the relevant group is the non-compact Lorentz group $\mathrm{SO}(3,1) \sim \mathrm{SL}(2, \mathbb{C})$. We expect the procedure proposed in this paper to extend directly, once the harmonic analysis for the Lorentz group is used. In particular, the Gaussian $\exp \left(-\sin ^{2} \phi\right)$ for $\mathrm{SU}(2)$ used in the boundary state should be replaced by the analogous $\exp \left(-\sinh ^{2} \phi\right)$ for $\operatorname{SL}(2, \mathbb{C})$, or maybe by the simpler Gaussian $\exp \left(-\phi^{2}\right)$. The latter case could further simplify the analysis of the group integrals.

\section{Acknowledgments}

We thank John Baez, Dan Christensen and Carlo Rovelli for useful discussions and encouragement. Research at Perimeter Institute is supported in part by the Government of Canada through NSERC and by the Province of Ontario through MEDT. 


\section{APPENDIX: GEOMETRY OF A 4-SIMPLEX}

Where more convenient, we use the double index notation for the links: $l \equiv I J$, where $I$ and $J$ are the two nodes linked by $l$.

Let us consider five $4 \mathrm{~d}$ unit vectors $\hat{N}_{I} \in \mathcal{S}^{3}, I=1 \ldots 5$, and introduce the ten angles defined by their scalar products, $\cos \phi_{I J}=\hat{N}_{I} \cdot \hat{N}_{J}$, with the convention $\phi_{I I}=0$. Finally, we define the $5 \times 5$ Gram matrix, $G_{I J}=\cos \phi_{I J}$. These five vectors are not linearly independent, so we can find $v_{I} \in \mathbb{R}^{5}$ such that:

$$
\sum_{I=1}^{5} v_{I} \hat{N}_{I}=0 .
$$

This means that the 5 -vector $v_{I}$ is a null vector for the Gram matrix $G_{I J}$. In particular, we get a constraint on the angles $\phi_{I J}$ :

$$
\forall J, \quad \sum_{I} v_{I} \hat{N}_{I} \cdot \hat{N}_{J}=0 \quad \Rightarrow \quad \operatorname{det} G_{I J}=0 .
$$

This constraint can be interpreted geometrically as follows. The five unit vectors define a unique geometric 4simplex (embedded in $\mathbb{R}^{4}$ ) up to a global scale (4-volume of the simplex). They are the (outward) normals to the five tetrahedra of the 4-simplex. The closure condition of the 4 -simplex reads exactly as (53) with the $v_{I}$ being the (oriented) 3 -volumes of the tetrahedra. Furthermore, we can differentiate the equation (54) and contract it with the null vector. This gives:

$$
\sum_{I, J} v_{I} v_{J} \sin \phi_{I J} \mathrm{~d} \phi_{I J}=0
$$

Next, recall the well known relation $3 v_{I} v_{J} \sin \phi_{I J}=4 \mathcal{V} A_{I J}$, where $\mathcal{V}$ is the 4 -volume of the simplex and $A_{I J}$ the area of the triangle shared by the two tetrahedra $I$ and $J$. This relation allows to write (55) in a simple geometric form as

$$
\sum_{I \neq J} A_{I J} \mathrm{~d} \phi_{I J}=0
$$

which is the Schläfli identity.

On the other hand, directly differentiating the $\operatorname{det} G_{I J}=0$ constraint gives:

$$
\sum_{I<J} \sin \phi_{I J} \Lambda^{(I J)} \mathrm{d} \phi_{I J}=0,
$$

where $\Lambda^{(I J)}$ is the off-diagonal minor obtained by removing the $I$ th line and $J$ th column from the Gram matrix $G_{I J}$. In particular, this means that the minors $\Lambda^{(I J)}$ are related to the areas $A_{I J}$, up a global scale factor $\kappa$ :

$$
\Lambda^{(I J)}=\kappa \frac{A_{I J}}{\sin \phi_{I J}} .
$$

This relation was used in Section II.

The minors $\Lambda^{(I J)} \equiv \Lambda^{(l)}$ play a major role in evaluating the components of the graviton propagator. In fact, their derivatives enter the second derivatives of the action (32). Using $\sin \overline{\phi_{l}}=\eta_{l} \sigma_{l} \sin \theta$ and $\Lambda^{(l)}\left(\overline{\phi_{l}}\right)=\sigma_{l} \Lambda_{0}$, $\Lambda_{0}:=\cos \theta(1-\cos \theta)^{3}$, at the saddle point (38) we have

$$
\left.\frac{\partial^{2} S}{\partial \phi_{l} \partial \phi_{l^{\prime}}}\right|_{\text {saddle }}=\frac{4}{\alpha} \cos 2\left(\phi_{l}-\eta_{l} \theta\right) \delta_{l l^{\prime}}+\left.2 i \frac{\kappa}{j_{0}}\left(\cos \phi_{l} \Lambda^{(l)} \delta_{l l^{\prime}}+\sin \phi_{l} \frac{\partial \Lambda^{(l)}}{\partial \phi_{l^{\prime}}}\right)\right|_{\text {saddle }} \equiv \eta_{l} \eta_{l^{\prime}} A_{l l^{\prime}}^{\epsilon}
$$

where

$$
A_{l l^{\prime}}^{\epsilon}:=\left(\frac{4}{\alpha}+2 i \epsilon \cot \theta\right) \delta_{l l^{\prime}}+\left.2 i \epsilon \frac{1}{\Lambda_{0}} \frac{\partial \Lambda^{(l)}}{\partial \phi_{l^{\prime}}}\right|_{\phi=\theta}
$$


and

$$
\left.\frac{\partial^{2} S}{\partial \phi_{l} \partial \kappa}\right|_{\text {saddle }}=\left.2 i \frac{1}{j_{0}} \sin \phi_{l} \Lambda^{(l)}\right|_{\text {saddle }}=2 i \frac{1}{j_{0}} \eta_{l} \sin \theta \Lambda_{0}
$$

Notice that $A_{l l^{\prime}}^{-} \equiv \overline{A_{l l^{\prime}}^{+}}$.

Evaluated at the saddle points, the derivatives of the minors have the following values,

$$
\left.\frac{\partial \Lambda^{(l)}}{\partial \phi_{l^{\prime}}}\right|_{\text {saddle }}=\left.\sigma_{l} \eta_{l} \frac{\partial \Lambda^{(l)}}{\partial \phi_{l^{\prime}}}\right|_{\phi=\theta}= \begin{cases}-\sigma_{l} \eta_{l} \Lambda_{0} \frac{2 \cos ^{2} \theta+3 \cos \theta+1}{\sin \theta \cos \theta} & \text { if } l=l^{\prime}, \\ \sigma_{l} \eta_{l} \Lambda_{0} \frac{1+\cos \theta}{\sin \theta} & \text { if } l \text { and } l^{\prime} \text { adjacient } \\ 0 & \text { if } l \text { and } l^{\prime} \text { opposite. }\end{cases}
$$

Using these and the explicit value $\theta=\arccos \left(-\frac{1}{4}\right)$ of the angle, (60) reads

$$
A_{l l^{\prime}}^{\epsilon}=\frac{6}{\sqrt{15}}\left(\frac{2 \sqrt{15}}{3 \alpha}-\frac{i}{3} \epsilon\right) \delta_{l l^{\prime}}+\frac{6}{\sqrt{15}} i \epsilon \begin{cases}2 & \text { if } l=l^{\prime} \\ 1 & \text { if } l \text { and } l^{\prime} \text { adjacient } \\ 0 & \text { if } l \text { and } l^{\prime} \text { opposite. }\end{cases}
$$

The matrix $A_{l l^{\prime}}:=A_{l l^{\prime}}^{+}$has only three independent entries, coming from the three independent components of the matrix $\frac{\partial \Lambda^{(l)}}{\partial \phi_{l^{\prime}}}$. Consequently, each row of the matrix and of its inverse shows the same structure, which is summarised in the table below. For convenience, we have introduced the quantity $\alpha^{\prime}=\frac{2 \sqrt{15}}{3 \alpha}$.

\begin{tabular}{|c||c||c|}
\hline matrix $A_{l l^{\prime}}$ & inverse matrix $\left(A^{-1}\right)_{l l^{\prime}}$ \\
$\frac{6}{\sqrt{15}}\left(\alpha^{\prime}+i \frac{5}{3}\right)$ & along the diagonal & $\frac{\sqrt{15}}{2} \frac{\left(3 \alpha^{\prime}+2 i\right)\left(3 \alpha^{\prime}+11 i\right)}{\left(3 \alpha^{\prime}+20 i\right)\left(-7 i+3 \alpha^{\prime}\right)\left(3 \alpha^{\prime}+5 i\right)}$ \\
$\frac{6}{\sqrt{15}} i$ & occuring six times & $-i \frac{3 \sqrt{15}}{2} \frac{\left(3 \alpha^{\prime}+11 i\right)}{\left(3 \alpha^{\prime}+20 i\right)\left(-7 i+3 \alpha^{\prime}\right)\left(3 \alpha^{\prime}+5 i\right)}$ \\
0 & occuring three times & $i \frac{3 \sqrt{15}\left(3 \alpha^{\prime}+11 i\right)}{\left(3 \alpha^{\prime}+20 i\right)\left(-7 i+3 \alpha^{\prime}\right)\left(3 \alpha^{\prime}+5 i\right)}$ \\
\hline
\end{tabular}

Finally, we have

$$
\operatorname{det} A_{l l^{\prime}}=\frac{1024}{759375}\left(3 \alpha^{\prime}+5 i\right)\left(3 \alpha^{\prime}+20 i\right)^{4}\left(-7 i+3 \alpha^{\prime}\right)^{5}
$$

and

$$
f_{1}(\alpha):=\sum_{l^{\prime}}\left(A^{-1}\right)_{l l^{\prime}} \equiv \frac{\sqrt{15}}{2\left(\frac{2 \sqrt{15}}{\alpha}+5 i\right)} \quad \forall l
$$

from which $\sum_{l l^{\prime}}\left(A^{-1}\right) l l^{\prime}=10 f_{1}(\alpha)$.

[1] C. Rovelli, "Graviton propagator from background-independent quantum gravity," Phys. Rev. Lett. 97 (2006) 151301 arXiv:gr-qc/0508124.

[2] L. Modesto and C. Rovelli, "Particle scattering in loop quantum gravity," Phys. Rev. Lett. 95 (2005) 191301 arXiv:gr-qc/0502036.

[3] S. Speziale, "Towards the graviton from spinfoams: The 3d toy model," JHEP 05 (2006) 039 arXiv:gr-qc/0512102. 
[4] E. Livine, S. Speziale and J. Willis "Towards the graviton from spinfoams: higher order corrections in the 3d toy model," arXiv:gr-qc/0605123

[5] E. Bianchi, L. Modesto, C. Rovelli and S. Speziale, "Graviton propagator in loop quantum gravity," Class. Quant. Grav. 23 (2006) 6989 arXiv:gr-qc/0604044.

[6] C. Rovelli. Quantum Gravity. (Cambridge University Press, Cambridge 2004.)

[7] J. W. Barrett and L. Crane, "Relativistic spin networks and quantum gravity," J. Math. Phys. 39, 3296 (1998) arXiv:gr-qc/9709028.

[8] J. W. Barrett, "The Classical evaluation of relativistic spin networks," Adv. Theor. Math. Phys. 2 (1998) 593 arXiv:math.qa/9803063.

L. Freidel and K. Krasnov, "Simple spin networks as Feynman graphs," J. Math. Phys. 41 (2000) 1681 arXiv:hep-th/9903192.

[9] C. Rovelli and S. Speziale, "A semiclassical tetrahedron," Class. Quant. Grav. 23 (2006) 5861 arXiv:gr-qc/0606074.

[10] F. Mattei, C. Rovelli, S. Speziale and M. Testa, "From 3-geometry transition amplitudes to graviton states," Nucl. Phys. B 739, 234 (2006) arXiv:gr-qc/0508007.

[11] J. W. Barrett and R. M. Williams, "The asymptotics of an amplitude for the 4-simplex", Adv.Theor.Math.Phys. 3 (1999) 209-215, arXiv:gr-qc/9809032;

J. W. Barrett and C. M. Steele, "Asymptotics of Relativistic Spin Networks", Class.Quant.Grav. 20 (2003) 1341-1362, arXiv:gr-qc/0209023

[12] L. Freidel and D. Louapre, "Asymptotics of $6 \mathrm{j}$ and $10 \mathrm{j}$ symbols," Class. Quant. Grav. 20 (2003) 1267 arXiv:hep-th/0209134.

[13] J.C. Baez, J.D. Christensen, G. Egan, "Asymptotics of 10j symbols", Class.Quant.Grav. 19 (2002) 6489, arXiv:gr-qc/0208010

[14] J. D. Christensen, "Numerical analysis of the graviton from the Barrett-Crane vertex," to appear.

[15] L. Freidel and E. R. Livine, "Ponzano-Regge model revisited. III: Feynman diagrams and effective field theory," arXiv:hep-th/0502106.

[16] E. R. Livine and S. Speziale, "Particle insertions and Geometrical Measurements in 3d Quantum Gravity," in preparation.

[17] E.R. Livine and D. Oriti, "Coupling of spacetime atoms and spin foam renormalisation from group field theory," arXiv:gr-qc/0512002. 